\title{
Food safety concerns of biofilm-forming zoonotic bacterial pathogens
}

\begin{abstract}
A. K. Bhunia ${ }^{1 *}$
${ }^{1}$ Molecular Food Microbiology Laboratory, Department of Food Science, Purdue Institute of Inflammation, Immunology and Infectious Disease, Department of Comparative Pathobiology, Purdue University, West Lafayette, Indiana 47907, USA

Abstract

Transmission of zoonotic pathogens from livestock to humans through contaminated meat, dairy, and egg products presents significant food safety and public health concerns. Bacterial pathogens form biofilms as an integral part of their life cycle. Biofilms also aid in microbial persistence and disease progression in livestock and become a major source for zoonotic infection. Zoonotic pathogens of food safety concern include but are not limited to Listeria monocytogenes, Staphylococcus aureus, Clostridium perfringens, Yersinia enterocolitica, Salmonella enterica, Campylobacter spp., and Escherichia coli. Several bacterial virulence factors are recognized to have dual functions in biofilm formation and pathogenicity. Implementing strategies to prevent and control biofilms through inactivation of signaling molecules, cyclicdi-GMP (guanosine monophosphate) and quorum sensing would be an effective robust approach for reducing zoonotic diseases, food safety and public health concerns.
\end{abstract}

Key words: Biofilm, Food safety, Livestock, Quorum sensing, Zoonotic pathogens

\section{Highlights}

- Biofilm helps in pathogen colonization, persistence and disease progression.

- Biofilms protect pathogens from antibiotics and immune cells.

- Several bacterial virulence factors have dual functions in biofilm formation and pathogenicity.

- Prevention and control of biofilms are effective approaches for reducing zoonotic infection and foodborne diseases.

\section{Introduction}

Bacterial biofilms, zoonotic pathogens and food safety: Zoonotic pathogens not only affect the health of livestock, but also humans due to their transmission via meat, dairy products, eggs, other animal by-products, and indirectly through fruits and vegetables due to environmental contamination. Zoonotic diseases also affect food security, economic loss, and farmers' livelihood (Gebreyes et al., 2020). Zoonotic pathogens of food safety importance include Listeria monocytogenes, Staphylococcus aureus, Clostridium perfringens, C. difficile, Mycobacterium paratuberculosis, Bacillus anthracis, Brucella abortus, Salmonella enterica, Escherichia coli, Yersinia enterocolitica, and Campylobacter spp. (Bhunia, 2018; Abebe et al., 2020; Gebreyes et al., 2020). These pathogens, in part, are responsible for approximately 2 billion illnesses and over one million deaths globally (Kirk et al., 2015) and about 48 million illnesses, and 3000 deaths in the United States (Scallan et al., 2011) annually. Therefore, understanding the pathophysiology of zoonotic diseases by biofilm-forming pathogens in livestock and their dissemination to food provides an opportunity to improve food safety and reduce human foodborne illnesses.

Bacterial biofilms are a well-structured protective microbial community that allows the microbial cell to resist any unfavorable threats, such as the immune system, antibiotics and

"Corresponding Author, E Mail: bhunia@purdue.edu 
antimicrobials in a host or food processing environments, such as sanitizers, desiccation, ultraviolet radiation, and metallic cations (Flemming and Wingender, 2010; Ren et al., 2015; Bai et al., 2021b). Steps involved in biofilm formation include bacterial attachment, microcolony formation, maturation and detachment or dispersion. Microorganisms express fimbriae, curli, flagella, adhesion proteins, and capsules to firmly attach to a surface to initiate and form biofilms (Flemming and Wingender, 2010; Nadell et al., 2016). In biofilms, bacteria release an extracellular polymeric substance (EPS) consisting of polysaccharides, proteins, and extracellular DNA (e-DNA) forming a three-dimensional biofilm scaffold (Nadell et al., 2016). Bacteria also secrete signaling molecules such as $3^{\prime}, 5^{\prime}$ cyclic di-guanosine monophosphate (cyclic-diGMP), and quorum sensing (QS) molecules, which regulate gene/protein expression necessary for biofilm formation and virulence (Cotter and Stibitz, 2007; Landini et al., 2010; Römling et al., 2013). QS molecules help bacterial communication with other cells (cellto-cell communication) when they grow in close proximity. The QS molecules include Nacyl homoserine lactone (AHL), autoinducer2 (AI-2), AI-3, autoinducing peptides (AIPs), indole, pyrones, quinolones and several other small molecules. The QS molecules help biofilm formation and also regulate gene expression for bacterial survival, growth, cell density, motility, resistance to antimicrobials, tolerance to desiccation, and pathogenesis (Riedel et al., 2009; Landini et al., 2010).

Regulating biofilm formation by manipulating cyclic-di-GMP and QS production is an intriguing approach in controlling infection and pathogenesis (Caly et al., 2015; Kim et al., 2017; Almeida et al., 2018; Aswathanarayan and Vittal, 2018; Defoirdt, 2018). In addition, natural antimicrobials, microorganisms, or nanotechnology approaches could be employed to inactivate microbes in biofilms to prevent pathogen transmission (Singh et al., 2018; Bhunia, 2019; Liu et al., 2021; Rao et al., 2021).
Biofilms can be comprised of single-species or mixed-bacterial species. Often, a strong biofilmforming strain can provide shelter to other pathogens including the weak biofilm former within the biofilm scaffold to promote pathogen survival and persistence. The mixed culture biofilm can also facilitate genetic exchange between bacteria which may often perpetuate the spread of antibiotic resistance (Madsen et al., 2012; Stalder and Top, 2016).

During infection, pathogens also form biofilms to aid in adhesion for colonization on biotic surfaces including feathers, skin, oral cavity, respiratory tract, intestine, and urogenital tract. Pathogens also form biofilms on abiotic surfaces such as floors, walls, slaughterhouse or dairy processing equipment, food-contacting surfaces and drains. This implies that pathogens must express molecules that can promote biofilm formation and persistence in the environment and host. Virulence factors that are involved in both biofilm formation and pathogenesis are discussed below for major zoonotic bacterial pathogens.

\section{Virulence factors involved in host cell interaction and biofilm formation by zoonotic bacterial pathogens \\ Listeria monocytogenes: Listeria mono-} cytogenes is a rod-shaped, Gram-positive, facultative intracellular pathogen and is ubiquitously distributed in nature. Improperly produced silage is a major source of L. monocytogenes in ruminants, while readyto-eat meat, dairy products, fruits and vegetables are the major source for humans. L. monocytogenes being an etiologic agent for mastitis in cows, milk is one of the major sources (Winter et al., 2004). The major route of pathogen transmission to humans and animals is oral. Therefore, the gastrointestinal phase of infection is central to its systemic dissemination (Drolia and Bhunia, 2019; LopesLuz et al., 2021). As a foodborne pathogen, crossing the intestinal epithelial barrier is a critical step for disease progression. 
L. monocytogenes uses three major invasive pathways: (a) Listeria adhesion protein (LAP), (b) Internalin A (InlA), and (c) M-cell-mediated translocation to cross the intestinal barrier (Drolia and Bhunia, 2019). It then systemically disseminates to the spleen, liver, mesenteric lymph node, gall bladder, brain, and placenta in pregnant women. Epithelial cell invasion and cell-to-cell movement allow bacteria to maintain an intracellular lifestyle aided by numerous virulence factors (Lopes-Luz et al., 2021). L. monocytogenes invades the central nervous system to cause meningitis and encephalitis as manifested by circling disease (due to the loss of balance and gait), common in ruminants. The bacterium also causes abortion, premature birth, and stillbirth in the ovine and humans. Three major virulence factors including actin polymerization protein (ActA), Listeria adhesion protein (LAP), and Teichoic acid are shown to play a central role in biofilm formation. ActA, regulated by PrfA facilitates bacterial cell-to-cell movement by rearranging host cell cytoskeletal proteins. It also promotes bacterial aggregation and biofilm formation on intestinal epithelial cells and aids in disease progression (Travier et al., 2013).

Listeria teichoic acid is a structural component of the cell wall (peptidoglycan) consisting of alternating phosphate and ribitol or glycerol. Teichoic acid helps bacterial attachment to the host cells and also contributes to biofilm formation in L. monocytogenes ( $\mathrm{Zhu}$ et al., 2018). Tunicamycin (antibiotic) inhibits teichoic acid biosynthesis, thus interferes with L. monocytogenes adhesion and invasion to intestinal epithelial cells and biofilm formation (Zhu et al., 2018).

The involvement of Listeria adhesion protein (LAP) (Drolia et al., 2018) in biofilm formation has been demonstrated in recombinant Lactobacillus casei expressing LAP from L. monocytogenes in a cultured cell model and mouse intestinal villi (Drolia et al., 2020). Though biofilm-forming L. monocytogenes has attenuated infectivity in a cell culture model, it is highly infective in a mouse model after oral dosing (Bai et al., 2021a) suggesting management of biofilm can potentially reduce the zoonotic spread of the disease.

Staphylococcus aureus: Staphylococcus aureus is a Gram-positive opportunistic pathogen and is commonly found in water, soil, animal mucus membrane, udder, anterior nares and on skin (Balasubramanian et al., 2017; Miao et al., 2017). It is also salt tolerant and as a commensal skin bacterium, often becomes a source of the contaminant in foods that are heavily handled. It also causes mastitis; hence milk and dairy products serve as a vehicle. Livestock-associated methicillin-resistant S. aureus (LA-MRSA) presents the major challenge due to its resistance to antibiotics. Antibiotic treatment for skin infection is under heightened scrutiny because of biofilm formation and widespread antibiotic resistance.

$S$. aureus is a strong biofilm former and helps bacterium to persist and cause chronic infection (Moormeier and Bayles, 2017; Di Domenico et al., 2019). A large number of surface molecules, collectively referred to as microbial surface components recognizing adhesive matrix molecules (MSCRAMMs) help bacterial adhesion and biofilm formation (Corrigan et al., 2007; Moormeier and Bayles, 2017). The major biomolecules that are specifically involved in both biofilm formation and host cell adhesion have redundant functions. Those include biofilm-associated protein (Bap), fibronectin-binding proteins (FnBP), S. aureus surface protein G (SasG), $S$. aureus protein A ( $\mathrm{SpA})$, cell wall-anchored clumping factor (ClfA), polysaccharide intercellular adhesin (PIA), and teichoic acids (TA) (Zapotoczna et al., 2016; Bai et al., 2021b).

Clostridium perfringens: Clostridium perfringens is a Gram-positive, rod-shaped, spore-forming, obligate anaerobe (aerotolerant) and inhabits human and animal intestines. This organism produces multiple toxins and is responsible for gas gangrenes, enteritis 
necroticans (pigbel), dysentery, enteritis, and enterotoxaemia in livestock. In humans, as a foodborne pathogen, it causes food poisoning, diarrhea, and antibiotic-associated diarrhea similar to Clostridium difficile. Biofilm formation and resistance to multiple antibiotics are important attributes of persistence and pathogenesis in livestock (Hu et al., 2018). C. perfringens Agr-like (CpAL) molecule is one of the major quorum-sensing molecules and regulates biofilm formation and toxin secretion (Vidal et al., 2015). Biofilm formation is aided by Type IV pili and CcpA protein. Furthermore, biofilm also protects cells from antibiotic and oxygen-induced damage (Varga et al., 2008).

Escherichia coli: E. coli is a Gram-negative, facultatively anaerobic commensal bacterium of the intestinal tract of animals and humans. Flagella, fimbriae or pili, and curli and lipopolysaccharides are important accessory molecules that help bacterial motility and colonization in the intestine. Only a few strains of E. coli strains are pathogenic, which are classified as enterotoxigenic E. coli (ETEC), enteropathogenic E. coli (EPEC), enteroaggregative $E$. coli (EAEC), diffusely adherent E. coli (DAEC), enteroinvasive E. coli (EIEC), adherent invasive E. coli (AIEC), and Shiga toxin-producing $E$. coli (STEC) that include enterohemorrhagic E. coli (EHEC) (Croxen et $a l ., 2013)$. Toxin production and epithelial adhesion, colonization are essential traits of pathogens. ETEC is responsible for piglet and calf diarrhea. Animals suffer from dehydration and electrolyte loss, which may be fatal. Survivors may experience poor growth. In humans, ETEC causes traveler's diarrhea, which may be fatal in severe cases. STEC strains, especially the EHEC strains can cause lethal foodborne diseases leading to hemorrhagic colitis and hemolytic uremic syndrome, often associated with kidney failure and death (Newell and La Ragione, 2018). Raw meat or meat products (Currie et al., 2019) and fresh produce (Yaron and Romling, 2014; de Oliveira Elias et al., 2019) are major sources of STEC.
E. coli form biofilms in the gastrointestinal tract employing adhesion molecules, and extracellular matrix including curli and cellulose (Rossi et al., 2018). Curli is consisted of amyloid protein nanofibers and is responsible for cell-to-cell and cell-to-surface attachment (Patel et al., 2011; Nguyen et al., 2014). Cellulose is the major exopolysaccharide consisting of (1-4)- $\beta$-linked linear glucose chain. Cellulose production is dependent on the activation of bacterial cellulose synthesis (BcsA) by cyclic-di-GMP (Römling et al., 2013). Some $E$. coli strains (EAEC) can form a thick aggregating biofilm (stacked-brick) using aggregative adherence fimbriae (AAF) on the intestinal mucosal surface and cause diarrhea (Boll et al., 2017).

Salmonella enterica: Salmonella enterica is a Gram-negative rod-shaped bacterium and has a broad host range infecting animals, poultry, and humans. There are over 2500 serovars of Salmonella enterica and some are hostspecific. Among the Salmonella enterica serovars, Enteritidis, Typhimurium, Newport, Heidelberg, Javiana, Dublin, and Choleraesuis are representative of commonly occurring zoonotic serovars (Boore et al., 2015; Snyder et al., 2019). These serovars cause severe foulsmelling diarrhea and septicemia in animals. Besides meat, milk, poultry, and eggs, Salmonellae also are transmitted via fresh produce, nuts, spices, and flours. Salmonellae infection in the ovary of laying hen is responsible for trans-ovarian transmission and contamination in eggs. Non-typhoidal Salmonella (NTS) also known as gastroenteritis-causing salmonellae are responsible for 93.8 million illnesses and 155,000 deaths in humans worldwide (Majowicz et al., 2010; Kirk et al., 2015). Salmonellae also form biofilms on human and animal intestines (Steenackers et al., 2012) and biofilm formation is facilitated by curli, flagella, Bap, cellulose, e-DNA, c-diGMP, and quorum sensing (Almeida et al., 2017; Harrell et al., 2020; Lories et al., 2020; Rana et al., 2021). c-di-GMP signaling network also supports the transition between virulence 
(invasion) and biofilm formation in Salmonella (Ahmad et al., 2011). The QS inhibitors have been considered potential therapeutics for Salmonella infection (Almeida et al., 2018; Aswathanarayan and Vittal, 2018)

Campylobacter spp.: Campylobacter sp. is a Gram-negative, curved or spiral (helical) rod. Its cork-screw motility is facilitated by a single polar flagellum. There are four major species; C. jejuni, C. coli, C. fetus, and C. lari. Campylobacter causes abortion in animals and as a zoonotic organism; it can be transmitted from animals to humans via food or contact. Poultry, eggs, raw milk are common sources of this organism. After ingestion, this organism invades and causes cell damage in the lower small intestine. It produces a cytolethal distending toxin (CDT) and the infection results in severe pain mimicking acute appendicitis. It also causes fever, diarrhea, meningitis, endocarditis, pancreatitis, and reactive arthritis. Sequelae of this disease are Guillain Barre's Syndrome, manifested by a paralytic neurological disorder. Campylobacter produces firm biofilms. Flagella, capsular polysaccharides, lipo-oligosaccharide, e-DNA, cellbinding proteins, Peb1A (CBF1), and Peb4 (CBF2) which assist in tissue adhesion and biofilm formation (Kim et al., 2015; Tram et al., 2020). Quorum sensing molecules including AI-2 and LuxSplay important role in biofilm formation (Plummer et al., 2011).

Yersinia spp.: The genus Yersinia is a Gramnegative coccoid rod and is considered a major zoonotic pathogen. Three major species including $Y$. enterocolitica, $Y$. pesudotuberculosis, and $Y$. pestis are important and share common $\mathrm{O}$ antigens. In addition, all three species also share Yersinia outer membrane proteins (YOPs), V (immunogenic protein), and $\mathrm{W}$ (nonprotective lipoprotein) antigens. $Y$. enterocolitica and $Y$. pseudotuberculosis are enteropathogenic and responsible for gastroenteritis and $Y$. pestis is responsible for the plague. Pig serves as the primary reservoir for $Y$. enterocolitica and $Y$. pesudotuberculosis while rodents and flea are for $Y$. pestis.

All three pathogens carry chromosomal and plasmid (pVY)-encoded virulence factors, which are required for adhesion, invasion, and colonization of intestinal epithelial cells and lymph nodes, growth inside macrophages, macrophage apoptosis, and serum resistance. The pYV plasmid-encoded virulence factors are also involved in biofilm formation (Wang et al., 2019). Flagella and exopolysaccharides are important factors regulated by RpoS in biofilm formation (Guan et al., 2015).

\section{Conclusions}

Zoonotic bacterial pathogens express several molecules that are essential for biofilm formation, quorum sensing, and adhesion to host cells and tissues for colonization. Many of these molecules have redundant functions. Therefore, in the absence of one or more molecules, bacteria still are capable of forming biofilm for colonization, persistence, and infection. Quorum sensing and c-di-GMP network are critical for biofilm formation. Hence, strategies to inhibit QS and c-di-GMP provide an opportunity for pathogen control in livestock and humans as an alternative to antibiotics.

Conflict of interest: Authors have no conflict of interest in this study.

\section{ACKNOWLEDGMENTS}

The research in the authors' laboratory is supported in part by a grant from the National Academy of Science (NAS) and USAID (AID263-A-15-00002), Korean Food Research Institute (KFRI grant \# E0142102-03), the U.S. Department of Agriculture, Agricultural Research Service, under Agreement No. 59-8072-6-001, and the USDA National Institute of Food and Agriculture (Hatch accession no. 1016249). Any opinions, findings, conclusions, or recommendations expressed in this publication are those of the author(s) and do not necessarily reflect the view of the USDA, USAID, or NAS. 


\section{REFERENCES}

Abebe E, Gugsa G and Ahmed M, 2020. Review on major food-borne zoonotic bacterial pathogens. J Trop Med, 2020: 4674235, doi: 10.1155/2020/ 4674235

Ahmad I, Lamprokostopoulou A, Le Guyon S, Streck E, Barthel Met al., 2011. Complex c-di-GMP signaling networks mediate transition between virulence properties and biofilm formation in Salmonella enterica serovar Typhimurium. PLoS One, 6(12): e28351, doi: 10.1371/journal.pone.0028351

Almeida F, de Pimentel-Filho N, Pinto U, Mantovani H, Oliveira L et al., 2017. Acyl homoserine lactonebased quorum sensing stimulates biofilm formation by Salmonella Enteritidis in anaerobic conditions. Arch Microbiol, 199(3): 475-486, doi: 10.1007/ s00203-016-1313-6

Almeida FAd, Vargas ELG, Carneiro DG, Pinto UM and Vanetti MCD, 2018. Virtual screening of plant compounds and nonsteroidal anti-inflammatory drugs for inhibition of quorum sensing and biofilm formation in Salmonella. Microb Pathog, 121: 369388, doi: 10.1016/j.micpath.2018.05.014

Aswathanarayan JB and Vittal RR, 2018. Inhibition of biofilm formation and quorum sensing mediated phenotypes by berberine in Pseudomonas aeruginosa and Salmonella Typhimurium. RSC Adv, 8(63): 36133-36141, doi: 10.1039/ C8RA06413J

Bai X, Liu D, Xu L, Tenguria S, Drolia R et al., 2021a. Biofilm-isolated Listeria monocytogenes exhibits reduced systemic dissemination at the early (1224 h) stage of infection in a mouse model. Npj Biofilms Microbiomes, 7(1): 18, doi: 10.1038/ s41522-021-00189-5

Bai X, Nakatsu CH and Bhunia AK, 2021b. Bacterial biofilms and their implications in pathogenesis and food safety. Foods, 10(9): 2117, doi: 10.3390/ foods 10092117

Balasubramanian D, Harper L, Shopsin B and Torres VJ, 2017. Staphylococcus aureus pathogenesis in diverse host environments. Pathog Dis, 75(1): fix005, doi: 10.1093/femspd/ftx005

Bhunia AK, 2018. Foodborne microbial pathogens: Mechanisms and pathogenesis. Springer

Bhunia AK, 2019. Microbes as a tool to defend against antibiotic resistance in food animal production. Indian J Anim Health, 58(2): 01-18, doi: 10.36062/ ijah.58.2SPL.2019.01-18

Boll EJ, Ayala-Lujan J, Szabady RL, Louissaint C, Smith RZ et al., 2017. Enteroaggregative Escherichia coli adherence fimbriae drive inflammatory cell recruitment via interactions with epithelial MUC1.
mBio, 8(3): doi: 10.1128/mBio.00717-17

Boore AL, Hoekstra RM, Iwamoto M, Fields PI, Bishop RD et al., 2015. Salmonella enterica infections in the United States and assessment of coefficients of variation: A novel approach to identify epidemiologic characteristics of individual serotypes, 1996-2011. PLoS One, 10(12): e0145416, doi: 10.1371/journal.pone.0145416

Caly DL, Bellini D, Walsh MA, Dow JM and Ryan RP, 2015. Targeting cyclic di-GMP signalling: A strategy to control biofilm formation? Curr Pharm Des, 21(1): 12-24, doi: 10.2174/ 1381612820666140905124701

Corrigan RM, Rigby D, Handley P and Foster TJ, 2007. The role of Staphylococcus aureus surface protein SasG in adherence and biofilm formation. Microbiology, 153(8): 2435-2446, doi: 10.1099/ mic.0.2007/006676-0

Cotter PA and Stibitz S, 2007. c-di-GMP-mediated regulation of virulence and biofilm formation. Curr Opin Microbiol, 10(1): 17-23, doi: 10.1016/ j.mib.2006.12.006

Croxen MA, Law RJ, Scholz R, Keeney KM, Wlodarska $\mathrm{M}$ et al., 2013. Recent advances in understanding enteric pathogenic Escherichia coli. Clin Microbiol Rev, 26(4): 822-880, doi: 10.1128/CMR.00022-13

Currie A, Honish L, Cutler J, Locas A, Lavoie M-C et al., 2019. Outbreak of Escherichia coli O157: $\mathrm{H} 7$ infections linked to mechanically tenderized beef and the largest beef recall in Canada, 2012. J Food Prot, 82(9): 1532-1538, doi: 10.4315/0362028X.JFP-19-005

de Oliveira Elias S, Noronha TB and Tondo EC, 2019. Salmonella spp. and Escherichia coli O157:H7 prevalence and levels on lettuce: A systematic review and meta-analysis. Food Microbiol, 84: 103217, doi: 10.1016/j.fm.2019.05.001

Defoirdt T, 2018. Quorum-sensing systems as targets for antivirulence therapy. Trends Microbiol, 26(4): 313-328, doi: 10.1016/j.tim.2017.10.005

Di Domenico EG, Cavallo I, Capitanio B, Ascenzioni F, Pimpinelli F et al., 2019. Staphylococcus aureus and the Cutaneous microbiota biofilms in the pathogenesis of atopic dermatitis. Microorganisms, 7(9): 301, doi: 10.3390/microorganisms7090301

Drolia R, Amalaradjou MAR, Ryan V, Tenguria S, Liu D et al., 2020. Receptor-targeted engineered probiotics mitigate lethal Listeria infection. Nat Commun, 11(1): 6344, doi: 10.1038/s41467-020-20200-5

Drolia R and Bhunia AK, 2019. Crossing the intestinal barrier via Listeria adhesion protein and internalin A. Trends Microbiol, 27(5): 408-425, doi: 
10.1016/j.tim.2018.12.007

Drolia R, Tenguria S, Durkes AC, Turner JR and Bhunia AK, 2018. Listeria adhesion protein induces intestinal epithelial barrier dysfunction for bacterial translocation. Cell Host Microbe, 23(4): 470-484, doi: 10.1016/j.chom.2018.03.004

Flemming $\mathrm{HC}$ and Wingender J, 2010. The biofilm matrix. Nat Rev Microbiol, 8(9): 623-633, doi: 10.1038/nrmicro2415

Gebreyes WA, Jackwood D, de Oliveira CJB, Lee C-W, Hoet AE et al., 2020. Molecular epidemiology of infectious zoonotic and livestock diseases. Microbiol Spectr, 8(2): 1-21, doi: 10.1128/ microbiolspec.AME-0011-2019

Guan J, Xiao X, Xu S, Gao F, Wang J et al., 2015. Roles of RpoS in Yersinia pseudotuberculosis stress survival, motility, biofilm formation and type VI secretion system expression. J Microbiol, 53(9): 633-642, doi: 10.1007/s12275-015-0099-6

Harrell JE, Hahn MM, D’Souza SJ, Vasicek EM, Sandala JL et al., 2020. Salmonella biofilm formation, chronic infection, and immunity within the intestine and hepatobiliary tract. Front Cell Infect Microbiol, 10: 624622, doi: 10.3389/fcimb.2020.624622

$\mathrm{Hu}$ W-S, Kim H and Koo OK, 2018. Molecular genotyping, biofilm formation and antibiotic resistance of enterotoxigenic Clostridium perfringens isolated from meat supplied to school cafeterias in South Korea. Anaerobe, 52: 115-121, doi: 10.1016/j.anaerobe.2018.06.011

Kim JS, Park C and Kim YJ, 2015. Role of flgA for flagellar biosynthesis and biofilm formation of Campylobacter jejuni NCTC11168. J Microbiol Biotechnol, 25(11): 1871-1879, doi: 10.4014/ jmb. 1504.04080

Kim MK, Zhao A, Wang A, Brown ZZ, Muir TW et al., 2017. Surface-attached molecules control Staphylococcus aureus quorum sensing and biofilm development. Nat Microbiol, 2: 17080, doi: 10.1038/nmicrobiol.2017.80

Kirk MD, Pires SM, Black RE, Caipo M, Crump JA et al., 2015. World Health Organization estimates of the global and regional disease burden of 22 foodborne bacterial, protozoal, and viral diseases, 2010: A data synthesis. PLoS Med, 12(12): e1001921, doi: 10.1371/journal.pmed.1001921

Landini P, Antoniani D, Burgess JG and Nijl R, 2010. Molecular mechanisms of compounds affecting bacterial biofilm formation and dispersal. Appl Microbiol Biotechnol, 86(3): 813-823, doi: 10.1007/s00253-010-2468-8

Liu Y, Wu L, Han J, Dong P, Luo X et al., 2021. Inhibition of biofilm formation and related gene expression of Listeria monocytogenes in response to four natural antimicrobial compounds and sodium hypochlorite. Front Microbiol, 11: 3523, doi: 10.3389/fmicb.2020.617473

Lopes-Luz L, Mendonça M, Fogaça MB, Kipnis A, Bhunia AK et al., 2021. Listeria monocytogenes: review of pathogenesis and virulence determinantstargeted immunological assays. Crit Rev Microbiol, 47(5): 647-666 doi: 10.1080/1040841X. 2021.1911930

Lories B, Belpaire TER, Yssel A, Ramon H and Steenackers HP, 2020. Agaric acid reduces Salmonella biofilm formation by inhibiting flagellar motility. Biofilm, 2: 100022, doi: 10.1016/ j.bioflm.2020.100022

Madsen JS, Burmølle M, Hansen LH and Sørensen SJ, 2012. The interconnection between biofilm formation and horizontal gene transfer. FEMS Immunol Med Microbiol, 65(2): 183-195, doi: 10.1111/j.1574-695X.2012.00960.x

Majowicz SE, Musto J, Scallan E, Angulo FJ, Kirk M et al., 2010. The global burden of nontyphoidal Salmonella gastroenteritis. Clin Infect Dis, 50(6): 882-889, doi: 10.1086/650733

Miao J, Liang Y, Chen L, Wang W, Wang J et al., 2017. Formation and development of Staphylococcus biofilm: with focus on food safety. J Food Saftey, 37(4): e12358, doi: 10.1111/jfs. 12358

Moormeier DE and Bayles KW, 2017. Staphylococcus aureus biofilm: A complex developmental organism. Mol Microbiol, 104(3): 365-376, doi: 10.1111/mmi.13634

Nadell CD, Drescher K and Foster KR, 2016. Spatial structure, cooperation and competition in biofilms. Nat Rev Microbiol, 14(9): 589-600, doi: 10.1038/ nrmicro.2016.84

Newell DG and La Ragione RM, 2018. Enterohaemorrhagic and other Shiga toxinproducing Escherichia coli (STEC): Where are we now regarding diagnostics and control strategies? Transbound Emerg Dis, 65 Suppl 1: 49-71, doi: 10.1111/tbed.12789

Nguyen PQ, Botyanszki Z, Tay PKR and Joshi NS, 2014. Programmable biofilm-based materials from engineered curli nanofibres. Nat Commun, 5(1): 110, doi: 10.1038/ncomms5945

Patel J, Sharma M and Ravishakar S, 2011. Effect of curli expression and hydrophobicity of Escherichia coli O157: H7 on attachment to fresh produce surfaces. J Appl Microbiol, 110(3): 737-745, doi: 10.1111/j.1365-2672.2010.04933.x

Plummer P, Zhu J, Akiba M, Pei D and Zhang Q, 2011. Identification of a key amino acid of LuxS involved 
in AI-2 production in Campylobacter jejuni. PLoS One, 6(1): e15876, doi: 10.1371/journal.pone. 0015876

Rana K, Nayak SR, Bihary A, Sahoo AK, Mohanty KC et al., 2021. Association of quorum sensing and biofilm formation with Salmonella virulence: story beyond gathering and cross-talk. Arch Microbiol, 203(10): 5887-5897, doi: 10.1007/s00203-02102594-y

Rao H, Choo S, Rajeswari Mahalingam SR, Adisuri DS, Madhavan P et al., 2021. Approaches for mitigating microbial biofilm-related drug resistance: A focus on micro-and nanotechnologies. Molecules, 26(7): 1870, doi: 10.3390/molecules 26071870

Ren D, Madsen JS, Sørensen SJ and Burmølle M, 2015. High prevalence of biofilm synergy among bacterial soil isolates in cocultures indicates bacterial interspecific cooperation. ISME J, 9(1): 81-89, doi: 10.1038/ismej.2014.96

Riedel CU, Monk IR, Casey PG, Waidmann MS, Gahan CGM et al., 2009. AgrD-dependent quorum sensing affects biofilm formation, invasion, virulence and global gene expression profiles in Listeria monocytogenes. Mol Microbiol, 71(5): 1177-1189, doi: $10.1111 / \mathrm{j} .1365-2958.2008 .06589 . \mathrm{x}$

Römling U, Galperin MY and Gomelsky M, 2013. Cyclic di-GMP: the first 25 years of a universal bacterial second messenger. Microbiol Mol Biol Rev, 77(1): 1-52, doi: 10.1128/MMBR.00043-12

Rossi E, Cimdins A, Lüthje P, Brauner A, Sjöling A et al., 2018. "It's a gut feeling" - Escherichia coli biofilm formation in the gastrointestinal tract environment. Crit Rev Microbiol, 44(1): 1-30, doi: 10.1080/1040841X.2017.1303660

Scallan E, Hoekstra RM, Angulo FJ, Tauxe RV, Widdowson MA et al., 2011. Foodborne illness acquired in the United States-major pathogens. Emerg Infect Dis, 17(1): 7-15, doi: 10.3201/ eid1701.P11101

Singh AK, Bai X, Amalaradjou MAR and Bhunia AK, 2018. Antilisterial and antibiofilm activities of pediocin and LAP functionalized gold nanoparticles. Front Sustain Food Syst, 2: 74, doi: 10.3389/ fsufs.2018.00074

Snyder TR, Boktor SW and M'Ikanatha NM, 2019. Salmonellosis outbreaks by food vehicle, serotype, season, and geographical location, United States, 1998 to 2015. J Food Prot, 82(7): 1191-1199, doi: 10.4315/0362-028X.JFP-18-494

Stalder T and Top E, 2016. Plasmid transfer in biofilms: a perspective on limitations and opportunities. NPJ
Biofilms Microbiomes, 2(1): 1-5, doi: 10.1038/ npjbiofilms.2016.22

Steenackers H, Hermans K, Vanderleyden J and de Keersmaecker SCJ, 2012. Salmonella biofilms: An overview on occurrence, structure, regulation and eradication. Food Res Int, 45(2): 502-531, doi: 10.1016/j.foodres.2011.01.038

Tram G, Day CJ and Korolik V, 2020. Bridging the gap: A role for Campylobacter jejuni biofilms. Microorganisms, 8(3): 452, doi: 10.3390/ microorganisms 8030452

Travier L, Guadagnini S, Gouin E, Dufour A, ChenalFrancisque V et al., 2013. ActA promotes Listeria monocytogenes aggregation, intestinal colonization and carriage. PLoS Pathog, 9(1): e1003131, doi: 10.1371/journal.ppat.1003131

Varga JJ, Therit B and Melville SB, 2008. Type IV pili and the CcpA protein are needed for maximal biofilm formation by the gram-positive anaerobic pathogen Clostridium perfringens. Infect Immun, 76(11): 4944-4951, doi: 10.1128/IAI.00692-08

Vidal JE, Shak JR and Canizalez-Roman A, 2015. The $\mathrm{CpAL}$ quorum sensing system regulates production of hemolysins CPA and PFO to build Clostridium perfringens biofilms. Infect Immun, 83(6): 24302442, doi: 10.1128/IAI.00240-15

Wang H, Palmer J and Flint S, 2019. Function of pYV plasmid on biofilm formation of Yersinia enterocolitica ERL032123 in the presence of Ca2+. J Food Prot, 82(10): 1683-1687, doi: 10.4315/0362028X.JFP-19-018

Winter P, Schilcher F, Bagò Z, Schoder D, Egerbacher $\mathrm{M}$ et al., 2004. Clinical and histopathological aspects of naturally occurring mastitis caused by Listeria monocytogenes in cattle and ewes. J Vet Med B Infect Dis Vet Public Health, 51(4): 176179, doi: 10.1111/j.1439-0450.2004.00751.x

Yaron S and Romling U, 2014. Biofilm formation by enteric pathogens and its role in plant colonization and persistence. Microb Biotechnol, 7(6): 496-516, doi: 10.1111/1751-7915.12186

Zapotoczna M, O’Neill E and O'Gara JP, 2016. Untangling the diverse and redundant mechanisms of Staphylococcus aureus biofilm formation. PLoS Pathog, 12(7): e1005671, doi: 10.1371/ journal.ppat.1005671

Zhu X, Liu D, Singh AK, Drolia R, Bai X et al., 2018. Tunicamycin mediated inhibition of wall teichoic acid affect Staphylococcus aureus and Listeria monocytogenes cell morphology, biofilm formation and virulence. Front Microbiol, 9: 1352, doi: 10.3389/fmicb.2018.01352

Received - 30.09.2021, Accepted - 10.11.2021, Published - 01.12.2021

Section Editors: Prof. S. Biswas and Dr. A. K. Das, Members, Editorial Board 